\title{
What are the potential biomarkers that should be considered in diagnosing and managing canine chronic inflammatory enteropathies?
}

\author{
Carina Sacoor, Luís Meireles Barros and Liliana Montezinho* \\ Center for Investigation Vasco da Gama (CIVG), University School of Vasco da Gama, Coimbra, Portugal
}

\begin{abstract}
Chronic inflammatory enteropathies in dogs are characterized by persistent or recurrent gastrointestinal signs that last for more than 3 weeks. Despite unclear etiopathogenesis, it is considered that a genetic predisposition associated with environmental factors, such as dietary antigens and intestinal microbiota, might induce an abnormal immune response in the host. The diagnosis of this condition requires full investigation in order to exclude all other possible causes. Currently, the observation of clinical signs associated with histopathologic evaluation and systematic therapeutic trials is the gold standard for the diagnosis of chronic enteropathies. Furthermore, diagnosis, monitoring the disease progression, and treatment response evaluation can be exhausting, since this whole process is time-consuming, costly, and partially invasive. Therefore, biomarkers appear as non-invasive tools, which can be useful in evaluating gastrointestinal function, identifying the presence of the disease and assessing its natural progression, monitoring gastrointestinal inflammation, predicting response to treatment, and clinical outcomes. Over the past decade, several studies were conducted in order to explore the clinical utility of biomarkers. Thus, the aim of this dissertation is to provide an overview of the biomarkers considered relevant in the diagnosis and management of dogs with chronic inflammatory enteropathies. The biomarkers addressed in this study may be serological, present in urine and feces, or even tissue-derived. This study argues that biomarkers, in particular calprotectin and calgranulin $\mathrm{C}$, have great potential to be used in clinical practice in the diagnosis and management of affected dogs. However, a single biomarker cannot assuredly predict disease severity, progression, response to treatment, and clinical outcomes. Therefore, in order to achieve greater accuracy, it would be beneficial if these tools are used in conjunction with contemporary ones. Future research is needed with the aim to better determine the usefulness of these tools in chronic inflammatory enteropathies in dogs.
\end{abstract}

Keywords: Biomarkers, Chronic enteropathies, Dog, Inflammatory bowel disease.

\section{Introduction}

Chronic inflammatory enteropathies (CIE) are a group of gastrointestinal (GI) disorders characterized by persistent or recurrent GI signs (Heilmann and Steiner, 2018; Moser et al. 2018), lasting for more than 3 weeks (AlShawaqfeh et al., 2017) with histological evidence of primary intestinal mucosal inflammation (Heilmann et al., 2018). There were inconsistencies in the articles pertaining to this research, as some authors used the term "chronic enteropathies" and others used the term "CIE". For the purpose of this study, the term "CIE" will be used.

The main cause of GI disease in dogs is considered to be CIE (Volkmann et al., 2017) which have a cyclical remission-relapse nature (Heilmann et al., 2016). They can be further classified in different forms, based on its clinical responsiveness to different therapeutic interventions (Heilmann et al., 2016), including food-responsive enteropathy (FRE), antibioticresponsive enteropathy (ARE), corticosteroid or immunosuppressive-responsive enteropathy (IRE), and non-responsive enteropathy (Dandrieux, 2016; Volkmann et al., 2017), if patients do not respond to immunomodulatory treatment (Heilmann and Steiner, 2018). In the cases of CIE that do not respond to food trials or antimicrobial treatments, therefore, requiring immunosuppressive-responsive treatment is also known as idiopathic inflammatory bowel disease (IBD) (Dandrieux, 2016; Volkmann et al., 2017). In addition to this classification, the term protein-losing enteropathy (PLE) is used when there is evidence of intestinal protein loss, revealing a worse prognosis and a poor clinical outcome (Dandrieux, 2016; Volkmann et al., 2017; Heilmann and Steiner, 2018).

While their exact etiologies and pathogenesis mechanisms remain partially uncertain, an abnormal immune response against dietary and bacterial antigens, associated with genetic predisposition, appears to play a central role (AlShawaqfeh et al., 2017; Hanifeh et al., 2018; Heilmann et al., 2018). Some breeds have been reported as predisposed for developing CIE, such as Weimaraner, Rottweiler, German Shepherd, Border Collie, Boxer (Dandrieux, 2016), Basenjis, and French bulldogs (Jergens et al., 2009)

The observation of clinical signs associated with histopathologic evaluation and systematic therapeutic 
trials is currently the gold standard for the diagnosis of CIE (Gerou-Ferriani et al., 2018). Histopathology allows the evaluation of the intestinal inflammatory infiltrate in the lamina propria. According to its nature, the inflammatory infiltrate can be divided into neutrophilic, eosinophilic, and lymphocyticplasmacytic, the latter described as the most frequent form of enteritis (AlShawaqfeh et al., 2017; Moser et al., 2018). Nevertheless, histopathology methods do not differentiate the various forms of CIE (Heilmann and Steiner, 2018). Hence, to date, the most accurate treatment is one of trial-and-error. Moreover, clinical outcomes for individuals vary widely and are difficult to predict (Gerou-Ferriani et al., 2018). As a result, novel biomarkers have been investigated in efforts to provide a more objective method to assess the natural progression of the disease, help in diagnostic evaluation, assess the temporal changes in clinical activity, patient monitorization, treatment evaluation, response, and outcome prediction (Im Hof et al., 2012; Jergens and Simpson, 2012; Gerou-Ferriani et al., 2018; Heilmann and Steiner, 2018; Otoni et al., 2018).

The aim of this study is to provide an overview of the current status of biomarkers and their usefulness in diagnosing and managing CIE in dogs. Another goal of this dissertation is to evaluate their potential clinical advantages, as well as possible limitations, based on the results of studies conducted mostly over the last decade.

\section{Etiopathogenesis}

Predisposed animals can develop CIE as a result of a dysregulation of mucosal immunity. The exact etiologies remain unknown and the underlying mechanisms of the pathogenesis have not been elucidated (Somu et al., 2017); however, one mechanism that could justify the development of chronic inflammation is the loss of immunologic tolerance against antigens, such as harmless dietary components and commensal microorganisms. The articles pertaining to this research believe that this failure of immunological tolerance occurs as a consequence of intestinal barrier integrity dysfunction (Eissa et al., 2019), dysregulation of gutassociated lymphoid tissue (GALT), disturbances in the bacterial flora, or a combination of these factors (Ogawa et al., 2018), resulting in pathological inflammations (Somu et al., 2017).

Intestinal epithelial cells (IECs) form a biochemical and physical barrier that separates luminal bacteria, dietary elements, toxins, and antigens from the host, preventing mucosal inflammation and tissue damage (Osada et al., 2016; Gram et al., 2018; Ogawa et al., 2018; Celi et al., 2019). Furthermore, IECs can secrete mucus and antimicrobial peptides in response to a stimulus (Eissa et al., 2019), contributing to epithelial repair and defending against bacterial invasion (Abraham and Cho, 2009). In CIE, the intestinal barrier has increased permeability as a result of a defective regulation of tight junctions and adherent junctions (Abraham and Cho, 2009; Ohta et al., 2014). A primary defect in barrier function can cause the above-mentioned abnormalities; however, those same abnormalities can be an outcome of inflammation (Abraham and Cho, 2009). Also, an impaired mucosal barrier function can result in increased exposure of immune cells to bacteria and intestinal luminal antigens, thus contributing to an unsuppressed immune response (Ohta et al., 2014). As such, IECs are essential in maintaining intestinal homeostasis (Osada et al., 2016).

A complex immunological network constitutes GALT (Junginger et al., 2012), which is composed of secondary lymphoid organs, including Peyer patches in the small intestine, isolated lymphoid follicles throughout the GI tract, the mesenteric lymph nodes, and effector sites, such as the lamina propria mucosa (Allenspach, 2011). The point of a framework like GALT is to promote tolerance toward environmental antigens, such as commensals and food antigens, while at the same time having a protective immune response against pathogens. Consequently, failure in maintaining this tolerance is the main factor leading to chronic intestinal inflammation (Allenspach, 2011; Gram et al., 2018). A complex population of innate and adaptive immune cells participates in the pathogenesis of CIE in dogs (Fig. 1) (Abraham and Cho, 2009; Allenspach, 2011; Eissa et al., 2019).

The innate immunity pathways provide an initial and rapid response (Abraham and Cho, 2009; Heilmann and Allenspach, 2017) and consist of IECs, neutrophils, dendritic cells (DCs), macrophages and eosinophils interaction, as well as their secreted products (Eissa et al., 2019).

Enterocyte pattern recognition receptors (PRRs) are responsible for the recognition of microbe-associated molecular patterns (Schnyder et al., 2018), which are conserved molecules found on bacteria or other infectious agents (Allenspach, 2011). Based on PRRs, one can determine whether the antigens are tolerated or reacted against (Cerquetella et al., 2010). PRRs include the nucleotide-binding oligomerization domain (NOD) 2 and toll-like receptors (TLRs), which are located on the surface or in the cytoplasm of IEC, DCs (Allenspach, 2011), and macrophages (Schnyder et al., 2018). TLRs, namely TLR-2, TLR-4, TLR5, and TLR-9, recognize specific bacterial products. To begin with, TLR-2 recognizes lipopeptides from Gram-positive bacteria; Secondly, TLR-4 identifies lipopolysaccharides from Gram-negative bacteria. Moreover, TLR-5 recognizes the main protein of bacteria flagella, flagellin (Hall, 2009; Allenspach, 2011; Heilmann and Allenspach, 2017); and finally, TLR-9 identifies bacterial and viral unmethylated CpG oligonucleotides (Schnyder et al., 2018). Also, NOD-2 detects the muramyl dipeptide molecule, a peptidoglycan component of Gram-positive and Gramnegative bacteria, and possibly viral constituents as well (Heilmann and Allenspach, 2017). Canine CIE have been linked with genetic polymorphisms in genes 


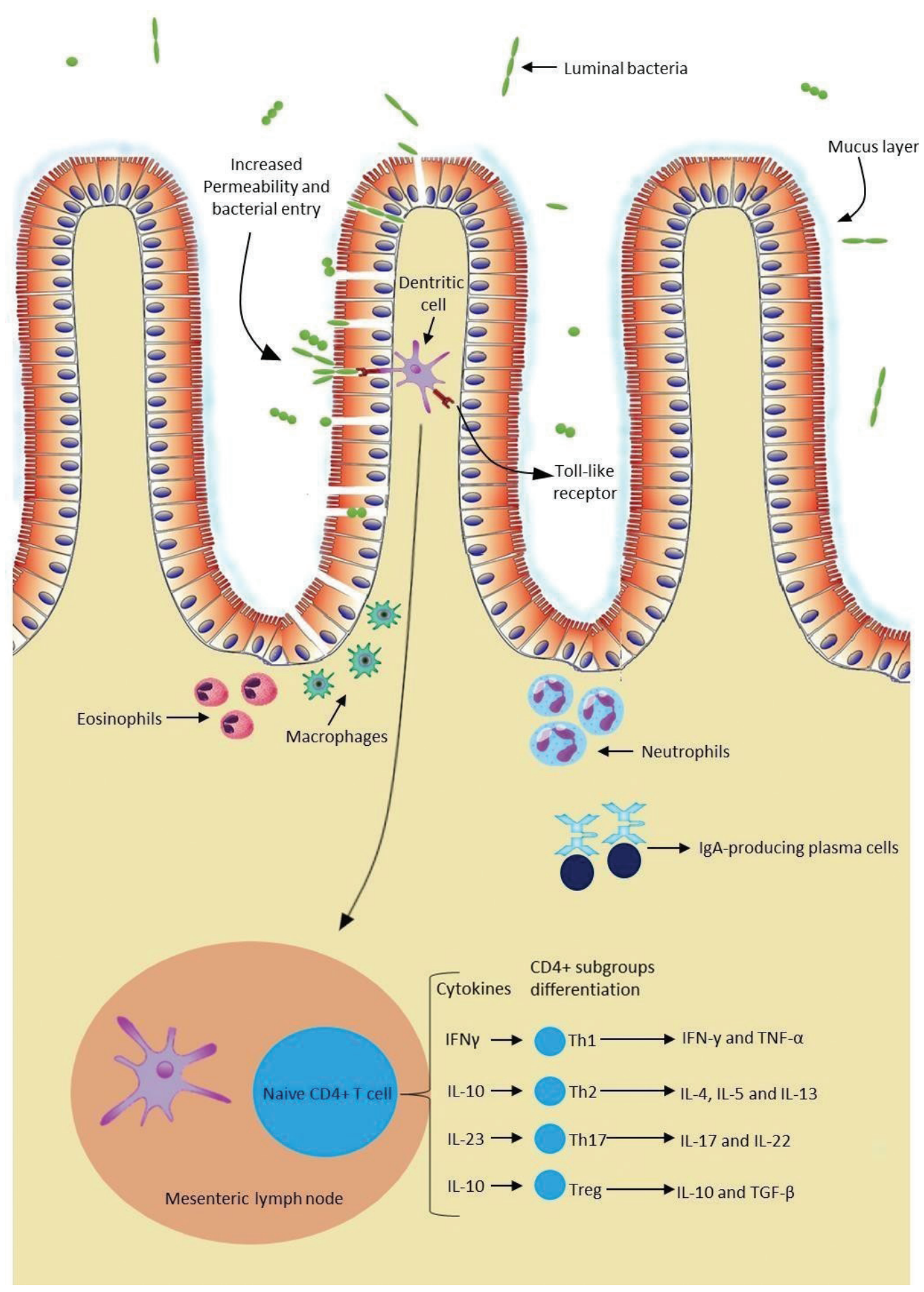

Fig. 1. The intestinal immune system in canine chronic inflammatory enteropathies. The intestinal barrier has increased permeability in dogs with CIE. An impaired barrier function can increase the exposure of immune cells to luminal antigens. These can be tolerated or reacted against, based on the PRRs, such as TLRs. Innate response is based on the interaction of IECs, neutrophils, macrophages, DCs, and eosinophils, as well as their secreted products. After innate immunologic mechanisms, activated APCs trigger an adaptive immune response by presenting peptide antigens to naïve CD4+ T helper cells in secondary lymphoid organs, such as the mesenteric lymph node. Based on their cytokine profile, Th cells can be differentiated into Th1, Th2, Th17 cells, and Tregs, and consequently produce their respective cytokines. In addition, intestinal B lymphocytes have the ability to turn into plasma cells and produce IgA antibodies, which contribute to immune protection. (Original illustration based on: Abraham and Cho, 2009; Allenspach, 2011; Eissa et al., 2019). 
encoding TLR2, TLR4, TLR5, and TLR9, which may contribute to individual predisposition (Maeda et al., 2012; Heilmann and Allenspach, 2017; Schnyder et al., 2018). These PRRs play an important role in the homeostasis and host defense. However, an abnormal activation of these PRRs can potentially lead to a loss of controlled homeostatic tolerance, causing chronic inflammations (Heilmann and Allenspach, 2017). After binding to TLRs, a complex intracellular signaling pathway is initiated (Allenspach, 2011), with the upregulation of pro-inflammatory cytokines, chemokines, costimulatory molecules, inflammatory mediators, such as prostaglandins and leukotrienes, reactive oxygen species, and nitrogen intermediates (Kołodziejska-Sawerska et al., 2013; Schnyder et al., 2018), culminating in the activation of nuclear factorkappa B (NF-kB) (Allenspach, 2011; Heilmann and Allenspach, 2017). In this changed environment, the immune system loses its tolerance, triggering an active immune response (Hall, 2009). Clinical studies have demonstrated that several innate immunity receptors, including TLR-2, TLR-4, TLR-5, TLR-9, and NOD-2, are dysregulated within the intestines of dogs suffering from CIE (Okanishi et al., 2013; Schnyder et al., 2018; Aono et al., 2019), thus representing consistent evidence that the innate immunity is hyperactive in this disease (Allenspach, 2011; Heilmann and Allenspach, 2017).

After innate immunologic mechanisms, activated antigen-presenting cells (APCs) trigger an adaptive immune response by presenting peptide antigens to naive $\mathrm{CD} 4+\mathrm{T}$ helper cells (Th cells) in secondary lymphoid organs. Based on their cytokine profile, Th cells can be differentiated into Th1 cells, which mediate cytotoxicity and cell-mediated immunity; Th2 cells, which mediate humoral immunity; and Th17 cells or regulatory T cells (Tregs) (Heilmann and Suchodolski, 2015). On top of that, memory lymphocytes are also developed (Abraham and Cho, 2009). In canine CIE, intestinal inflammation can be marked by Th1 responses, which are mediated mainly by the secretion of interferon gamma and tumor necrosis factor alpha. Th1 cells can be antagonized by Th2 cells that primarily produce interleukin (IL)-4, IL-5, and IL- 13, and are typically associated with responses to allergens and parasites (Eissa et al., 2019). Other studies have not been able to demonstrate a clear Th1 or Th2 cytokine expression in dogs with CIE (Jergens et al., 2009; Kołodziejska-Sawerska et al., 2013; Dumusc et al., 2014; Heilmann and Suchodolski, 2015; Eissa et al., 2019). Experts in the field observed that IL23 p19 is increased in the inflamed intestinal mucosa of dogs. This cytokine is produced by macrophages and plays an important role in the promotion of Th17 cell differentiation (Tamura et al., 2014). Th17 cells can produce proinflammatory cytokines, such as IL-17 and IL-22, but most importantly they can demonstrate anti-inflammatory properties due to their ability to transdifferentiate into Tregs. These cells play an important role in maintaining immunotolerance. They are in charge of suppressing effector T cells and APCs through the secretion of immunosuppressive cytokines, such as IL-10 and transforming growth factor beta (Maeda et al., 2016; Heilmann and Steiner, 2018; Eissa et al., 2019).

In dogs with CIE, this imbalance between proinflammatory and anti-inflammatory cytokines results in disrupted intestinal immunity (KołodziejskaSawerska et al., 2013). Although Th1, Th2, and Th17 are crucial for the defense against pathogens and elevated intake of luminal bacteria, their extension and overactivity can result in intestinal inflammation (Abraham and Cho, 2009).

In addition, intestinal B lymphocytes have the ability to turn into plasma cells and produce immunoglobulin (Ig) A antibodies, which contribute to immune protection without causing inflammation (Abraham and Cho, 2009). IgA antibodies not only contribute to the prevention of bacteria crossing the epithelial barrier, but can also shape the intestinal microbiota composition (Maeda et al., 2013), keeping them from triggering an immune response in the intestine (Lee et al., 2015).

\section{Current diagnostic challenges}

The diagnosis of CIE requires a complete investigation in order to exclude other possible causes of GI signs (Heilmann and Steiner, 2018; Moser et al., 2018), including infectious, neoplastic, metabolic, or endocrine diseases (Moser et al., 2018). This includes a detailed medical history, clinical examination, complete blood cell count, serum biochemical analyses, parasitological and bacteriologic fecal analyses, pancreatic function tests, medical imaging, including radiography and abdominal ultrasonography, which give information about intestinal layering and wall thickness. Furthermore, to acquire more specific information on the intestinal inflammation, an endoscopic evaluation with intestinal biopsies and histopathological evaluation, which distinguishes the various subtypes of mucosal infiltration, should be conducted (Cerquetella et al., 2010; Wdowiak et al., 2013; Allenspach, 2015; Moser et al., 2018). The interpretation of the obtained biopsies contributes to the assessment of the severity and distribution of the disease (Moser et al., 2018).

Clinical signs result from uncontrolled inflammation (Dandrieux, 2016) and generally include abdominal pain, vomiting, diarrhea, anorexia, weight loss, flatulence, bloating (Eissa et al., 2019), inappetence, and borborygmi (Kalenyak et al., 2018). Important tools for clinical evaluation are the canine IBD activity index (CIBDAI) and the canine chronic enteropathy clinical activity index (CCECAI) (Cerquetella et al., 2010). The former evaluates six parameters including attitude/ activity, appetite, vomiting, stool consistency, stool 


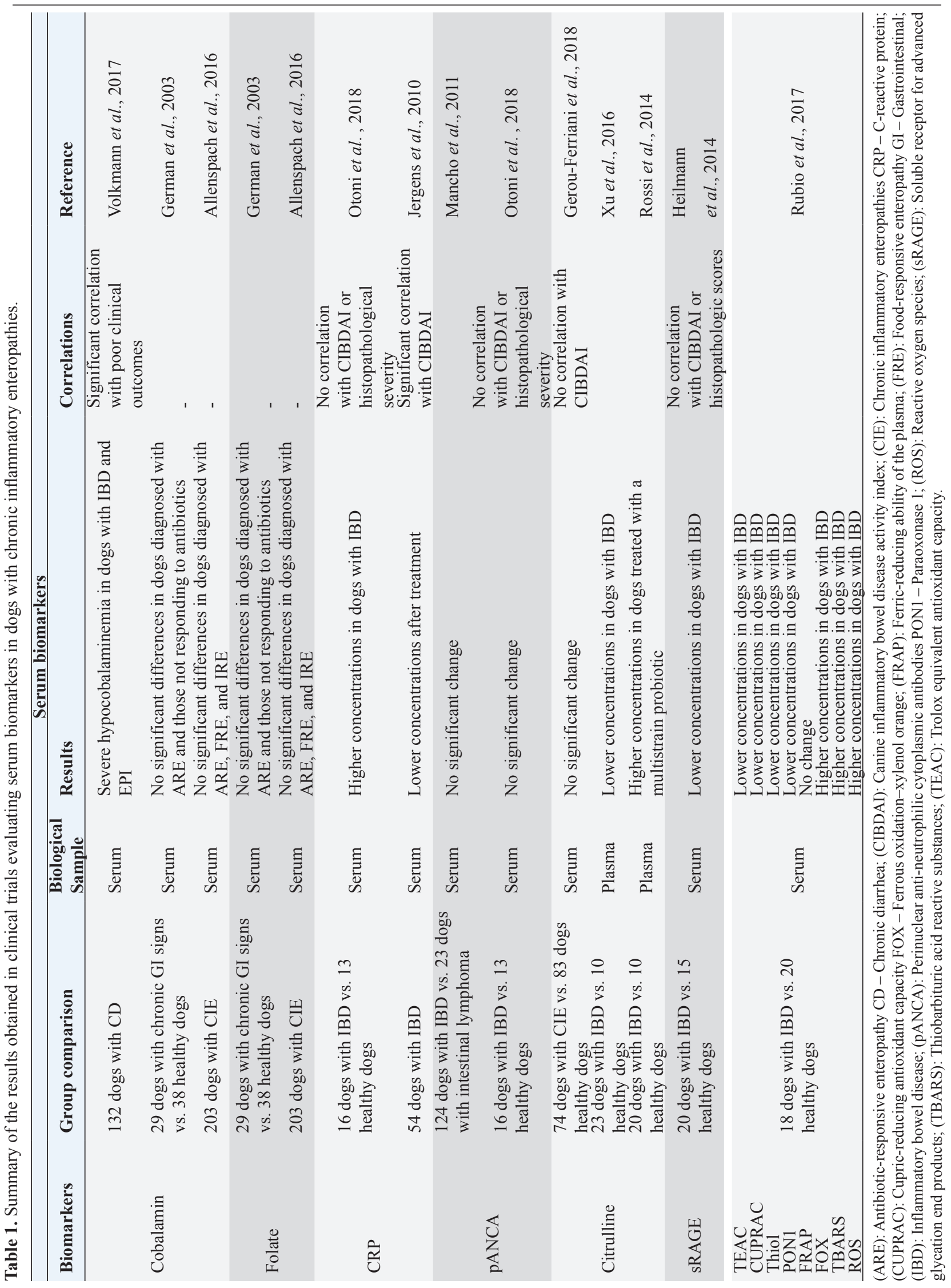




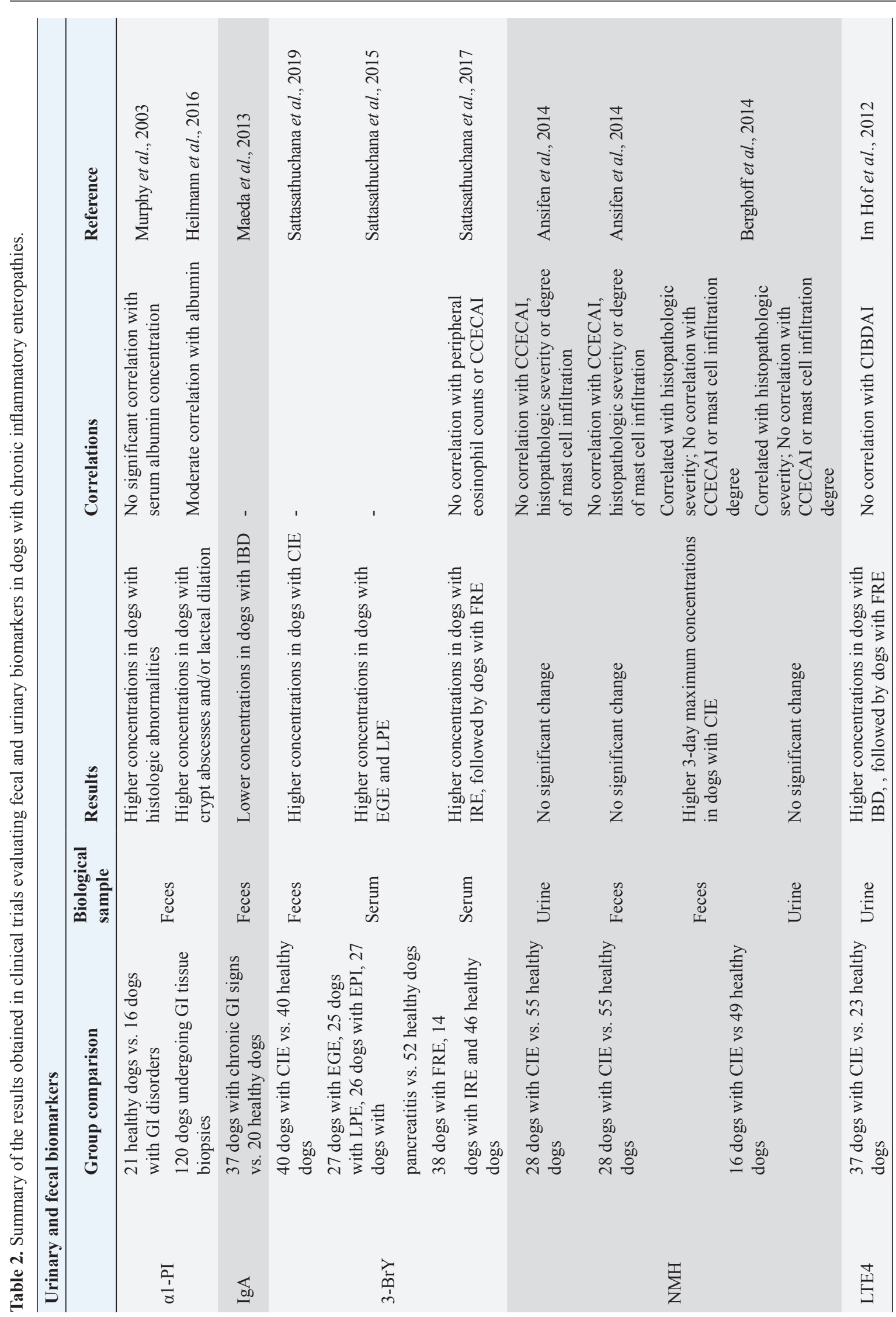




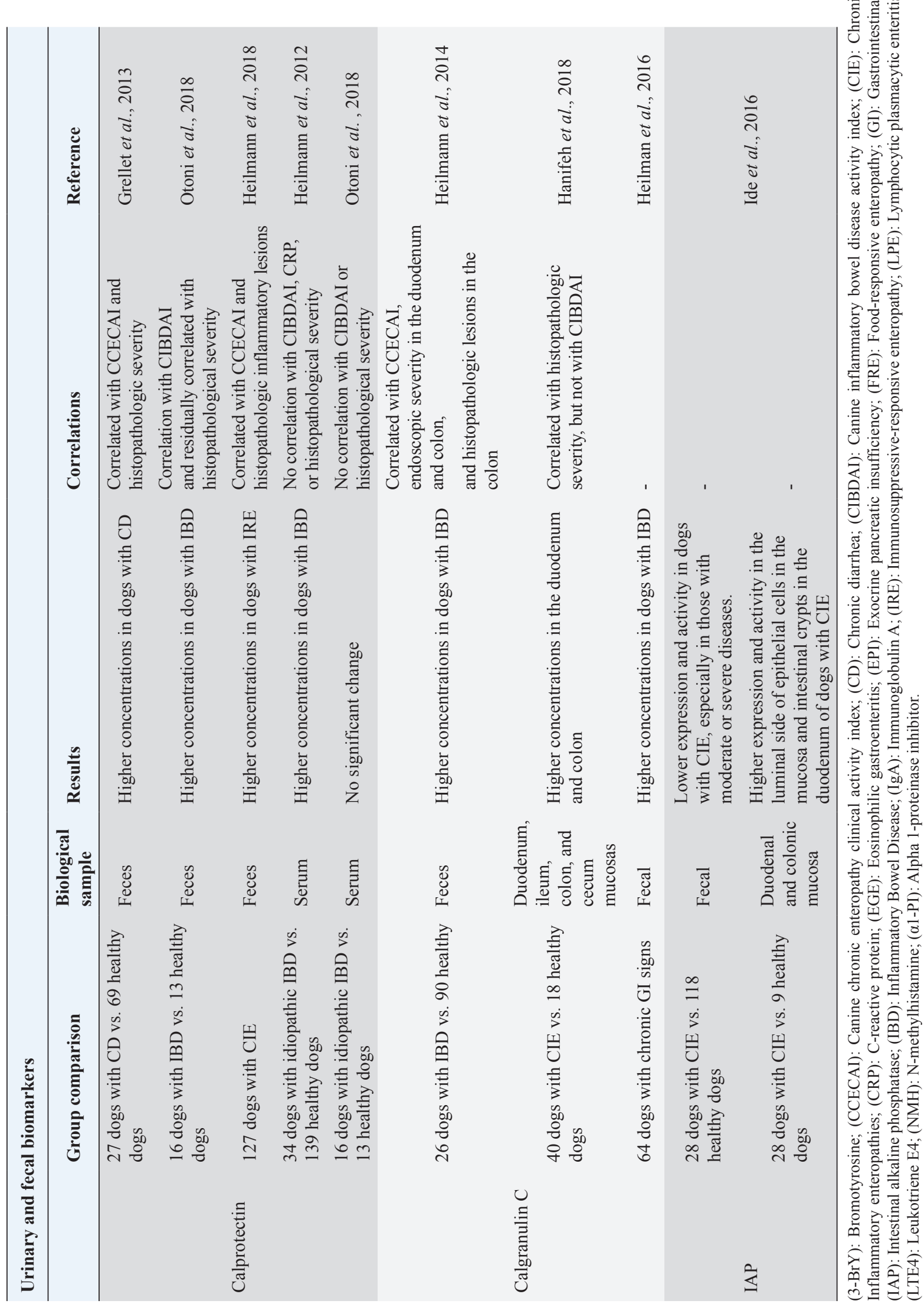


frequency, and weight loss. Each variable is scored from 0 (normal) to 3 (severe change). Based on a cumulative score, it classifies the disease as insignificant (0-3), mild (4-5), moderate $(6-8)$, or severe $(\geq 9)$ (Jergens et al., 2003). The above-mentioned clinical signs are also considered in CCECAI, together with albumin concentration, the presence of ascites, peripheral edema and pruritus. Based on a similar scoring pattern, CCECAI classifies the disease as insignificant (0-3), mild (4-5), moderate (6-8), severe (9-11), and very severe $(\geq 12)$ (Allenspach et al., 2007). However, these scoring systems only allow a semi-objective assessment of clinical disease activity (Heilmann et al., 2018). Typically, in clinical practice, veterinarians rely mainly on the severity of the clinical signs to estimate the disease severity and the response to treatment (Collins, 2013; Grellet et al., 2013). This evaluation is based on partially subjective assessments (Im Hof et al., 2012; Grellet et al., 2013), and additionally the severity of clinical signs has no proven correlation with the severity of histologic lesions (Heilmann et al., 2014, 2018), hence not reflecting intestinal inflammation (Collins, 2013).

It is worth noting that the endoscopic evaluation of the intestinal mucosa and histopathologic findings are usually not sufficient in differentiating the various forms of CIE (Heilmann and Steiner, 2018). However, these tools remain the golden standard for detecting and quantifying intestinal inflammation (Collins, 2013). Endoscopy procedures are costly, timeconsuming (Heilmann et al., 2014), and influenced by operator experience. Additionally, these are relatively invasive procedures (Otoni et al., 2018) that require general anesthesia, and the preparation of the colon. Histopathological examination of GI inflammation might be considered as limited, as clinical outcomes can be influenced by several factors. Among those are biopsy procedure, number of tissues samples, biopsy sample quality, and the diverse interpretations of GI histopathological findings among pathologists (Day et al., 2008; Simpson and Jergens, 2011; Wdowiak et al., 2013; Allenspach et al., 2018). The World Small Animal Veterinary Association (WSAVA) GI Standardization Group developed a grading scheme about histopathological standards for the characterization of inflammatory and associated morphological abnormalities in the canine and feline GI tract, in order to reduce fluctuations among interpretations (Day et al., 2008). However, even with the use of the WSAVA's standardization grading scheme, significant interobserver variability in the diagnostic interpretation of endoscopic mucosal specimens still exists (Allenspach, 2015; Allenspach et al., 2018), as well as lack of consensus (Simpson and Jergens, 2011). Another limitation to consider is the fact that improvement of the histopathologic lesions does not always correspond to the response to therapy and clinical improvement (Collins, 2013; Heilmann et al.,
2016). These procedures are unlikely to be frequently carried out (Heilmann et al., 2014). Furthermore, there are no currently available systems to accurately assess the degree of active inflammation.

Considering all these constraints, there is a need for a simple, minimally or non-invasive, and objective method that evaluates intestinal inflammation (Collins, 2013). Thus, biological markers for clinical indices, which objectively reflect mucosal disease severity, might be useful in clinical practice in diagnosing and managing GI inflammation (Heilmann et al., 2014; Otoni et al., 2018). It could be considered an attractive option for estimating a diagnosis, prognosis, and defining disease severity (Wdowiak et al., 2013; Otoni et al., 2018).

\section{Clinical relevance}

According to the European Commission Health Research Directorate (2010), "A biomarker is a biological characteristic, which can be molecular, anatomic, physiologic, or biochemical. These characteristics can be measured and evaluated objectively. They act as indicators of a normal or a pathogenic biological process. A biomarker shows a specific physical trait or a measurable biologically produced change in the body that is linked to a disease or a particular health condition". In CIE, biomarkers can be very useful tools in identifying the presence of disease, site of origin, evaluating the GI function, determining the progression of the disease, as well as the current response to treatment, and monitoring the severity of GI inflammation.

In the past decade, diverse biomarkers have been evaluated in dogs with CIE. In clinical practice, a useful biomarker should have the characteristics that make it valuable (Heilmann and Steiner, 2018). In order to have an added value, a biomarker should aim to be measurable without temporal delay in expression or secretion, specific to the disease process (Heilmann et al., 2014), easy to perform, have the ability to accurately identify individuals at risk (Jergens and Simpson, 2012), affordable, and minimally invasive, as well as being stable in routine biological samples (Heilmann and Steiner, 2018). In clinical practice, when using a single biomarker, it is essential to understand that it is improbable to meet all the criteria. Hence, clinical information about a specific biomarker should be taken into account for a better understanding of the data in a specific clinical situation (Heilmann and Steiner, 2018). The biomarkers addressed in this study may be serological, present in urine and feces, or even tissue-derived (Wdowiak et al., 2013). A summary of the clinical trials findings is presented in Tables 1 and 2.

\section{Biomarkers in chronic inflammatory enteropathies} Serological biomarkers

Cobalamin and folate concentrations. Cobalamin (vitamin B12) and folate (vitamin B9) are watersoluble vitamins (Heilmann and Steiner, 2018) of diagnostic and therapeutic importance (Collins, 2013). Most commercial pet foods usually contain cobalamin 
and folate, thus a dietary deficiency is very uncommon (Berghoff and Steiner, 2011). Some breeds, such as Chinese Shar Peis, Giant Schnanuzers, Border Collies, and Beagles, have cobalamin deficiency. Besides genetic predisposition, other causes for cobalamin deficiency in dogs are CIE and EPI (Toresson et al., 2016).

Hypocobalaminemia is more likely to occur due to disturbances in its absorptive mechanism. In the diet, cobalamin is bound to animal protein, preventing it from being absorbed. In the stomach, protein is partly digested. As a result, cobalamin is released and immediately binds to the R-binding protein. When entering the small intestine, pancreatic proteases breakdown the R-binding protein and the liberated cobalamin has high affinity to the intrinsic factor (IF), which is mainly secreted by the pancreas. After binding to IF, this complex is later absorbed into the ileum, the distal part of the small intestine by specific receptors (Berghoff and Steiner, 2011; Toresson et al., 2016).

Several factors can disturb this mechanism, resulting in cobalamin malabsorption. Chronic inflammation of the ileal mucosa can cause a reduced expression of the cobalamin-IF receptors in enterocytes (Berghoff and Steiner, 2011). Furthermore, as the main source of IF in dogs is the exocrine pancreas, its condition can also influence the binding nature with IF, and consequently affect cobalamin absorption (Toresson et al., 2016). Also, exocrine pancreatic insufficiency (EPI) may inhibit cobalamin dissociation from R-binding proteins (Berghoff and Steiner, 2011), disturbing the absorptive mechanism of cobalamin. Besides distal small intestinal malabsorption, hypocobalaminemia can also occur due to bacterial overgrowth in the small intestine, as cobalamin coupled with IF can be highly consumed by anaerobic intestinal bacteria (Berghoff and Steiner, 2011; Toresson et al., 2016; Moser et al., 2018). Similarly, Volkmann et al. (2017) identified the most severe decrease in serum cobalamin concentrations in dogs with IBD and EPI. A recent study showed hypocobalaminemia in $30 \%$ of dogs diagnosed with CIE (Heilmann et al., 2018). This condition is a negative prognostic factor in dogs with CIE, and can result in severe metabolic consequences (Toresson et al., 2016) and increased risk of euthanasia (Allenspach, 2015).

Hypocobalaminemia is not specific for CIE (Heilmann and Steiner, 2018). Similarly, no significant differences were observed in serum cobalamin levels between dogs diagnosed with ARE and those not responding to antibiotic treatment, or with other causes of chronic GI signs (German et al., 2003). Therefore, the measurement of serum cobalamin concentrations is not sufficient to differentiate the various forms of CIE, as demonstrated by Allenspach et al. (2016). However, a normal serum cobalamin concentration does not exclude a CIE diagnosis (Heilmann and Steiner, 2018), since the patient's body stores of cobalamin might still be sufficient to maintain a normal serum cobalamin concentration, despite malabsorption. Concentrations under the reference range require supplementation that should only be dropped if the underlying condition is fully resolved and when the patient's cobalamin concentrations is within the normal range values (Berghoff and Steiner, 2011).

Similar to cobalamin, alterations in serum folate concentrations are more likely to occur due to a reduced absorption, or alterations in intestinal microbiota (Berghoff and Steiner, 2011). Contrarily to cobalamin, folate is principally absorbed in the proximal part of the small intestine, the duodenum, and proximal jejune (Heilmann and Steiner, 2018). Dietary folate present in the form of folate polyglutamate is hydrolyzed by folate conjugase, an enzyme produced by the jejunal brush border (Berghoff and Steiner, 2011). Folate is then absorbed into the proximal part of the small intestine in the form of folate monoglutamate, by specific folate carriers (Heilmann and Steiner, 2018). When the proximal small intestinal mucosa is damaged, malabsorption of folate can occur due to an impaired folate conjugase activity, making folate unabsorbable, or even due to damaged folate carriers. Thus, hypofolatemia can occur if the condition has become chronic and the folate body stores have become depleted. Furthermore, due to dysbiosis in the small intestine, some intestinal bacteria can increase its folate production, becoming available for absorption by the host, resulting in false, normal, or higher serum concentrations (Berghoff and Steiner, 2011).

In a study carried out by Heilmann et al. (2018), hypofolatemia was shown in $14 \%$ and hyperfolatemia was shown in $5 \%$ of dogs diagnosed with CIE. Yet, even though hypofolatemia can result from chronic malabsorption in dogs with CIE, it is not specific for this condition. Researchers observed no significant differences in serum folate concentrations in dogs with CIE responsive to diet, antibiotic or immunosuppressive treatment (Allenspach et al., 2016). Moreover, German et al. (2003) observed insignificant differences in serum folate concentrations between dogs diagnosed with ARE and those not responding to antibiotic treatment or with other causes of chronic GI signs. Moreover, a normal serum folate concentration does not rule out a CIE diagnosis (Heilmann and Steiner, 2018).

Folate and cobalamin serum concentrations have been reported as nonspecific findings; nevertheless, its supplementation is important during treatment (Cerquetella et al., 2010).

C-reactive protein. C-reactive protein (CRP) is a positive type II acute phase protein of the pentraxin family. It is produced in the liver as a response to IL6 , IL-1 $\beta$, and tumor necrosis factor alpha (Jergens et al., 2009) during periods of infection, inflammation, or cancer (Heilmann et al., 2018).

Researchers showed increased CRP concentrations in dogs with idiopathic IBD, when compared to healthy 
dogs, and diseased dogs after treatment (Otoni et al., 2018). Some studies also noted a lack of correlation with clinical severity, as determined by CIBDAI, as well as with histopathologic lesions. Interestingly, in a larger study, a significant correlation between serum CRP concentrations and clinical severity, as determined by CIBDAI, was reported (Jergens et al., 2010).

Despite being considered a sensitive indicator of inflammation (Otoni et al., 2018), increased levels should be interpreted with caution (Allenspach, 2015) since CRP is not specific to the intestinal tract (Berghoff and Steiner, 2011) and can be increased in other diseases (Allenspach, 2015). Thus, this biomarker has limited utility as a diagnostic biomarker for CIE in dogs due to its high biological variability (Heilmann et al., 2018); Notwithstanding, it is valuable in other aspects such as monitoring treatment response and disease progression (Collins, 2013; Heilmann and Steiner, 2018).

Perinuclear antineutrophilic cytoplasmic antibodies . Perinuclear antineutrophilic cytoplasmic antibodies (pANCA) are serum autoantibodies directed toward neutrophil granule components (Mancho et al., 2010; Heilmann and Steiner, 2018), including nuclear histone, proteinase 3, myeloperoxidase (Heilmann and Steiner, 2018), lactoferrin, elastase, and lysozyme (Wdowiak et al., 2013). These antineutrophilic cytoplasmic antibodies can be detected by indirect immunofluorescence methods (Mancho et al., 2010; Heilmann and Steiner, 2018) through the visualization of a typical perinuclear staining pattern (Allenspach, 2015).

In general, canine antibodies might cross-react with diverse antigens, possibly resulting in the development of an autoimmune reaction that could be on the basis of chronic inflammation in dogs with IBD (Mancho et al., 2011). Thus, the detection of this biomarker has been proposed as a complementary tool to help in differentiating dogs with IBD from dogs with other chronic GI diseases (Mancho et al., 2011). The seropositivity of pANCA can also be detected in other infectious, inflammatory, autoimmune, or oncologic disorders, as it is non-CIE specific (Allenspach, 2015; Heilmann and Steiner, 2018). Similarly, Mancho et al. (2011) found no significant differences in pANCA expression between dogs with IBD and dogs with intestinal lymphoma.

With regard to intestinal inflammation, pANCA has not shown significant utility (Otoni et al., 2018). Furthermore, researchers failed to correlate pANCA seropositivity with CIBDAI and histopathologic scores (Otoni et al., 2018). Other authors notified the importance of pANCA in differentiating dogs with FRE and IBD (Allenspach, 2015). Dogs responding to food trials yielded more positive results, as cited in Otoni et al.'s (2018) study.

Although pANCA's utility has been considered as limited, it might be useful in differentiating various forms of CIE, as cited in Otoni et al.'s (2018) study.
Future studies should be carried out in order to evaluate the potential of pANCA as a useful biomarker in canine CIE.

Citrulline. Citrulline is a non-dietary amino acid produced and released by the enterocytes of the small intestinal mucosa (Xu et al., 2016; Gerou-Ferriani et al., 2018). Citrulline is not available in food; therefore, its serum concentration depends exclusively on the production by small intestine mucosa enterocytes. Similarly, a reduced serum concentration corresponds to a reduced enterocyte mass and absorptive function (Gerou-Ferriani et al., 2018). The authors suggested that citrulline has the potential to be an effective biomarker in chronic intestinal diseases in dogs. However, this study failed to show differences in serum citrulline concentrations between dogs with CIE and healthy dogs, as well as among dogs with different forms of CIE (Gerou-Ferriani et al., 2018). Furthermore, researchers observed failure not only in predicting treatment response, but also in correlating citrulline concentrations with disease severity, as determined by CIBDAI (Gerou-Ferriani et al., 2018). These unexpected findings could be explained by an insufficiently damaged enterocyte, which in turn would make a reduction in serum citrulline concentration undetectable (Gerou-Ferriani et al., 2018). Conversely, others observed, in a smaller study, a lower concentration of plasma citrulline in dogs diagnosed with IBD (Xu et al., 2016). Moreover, Rossi et al. (2014) reported a significant increase in plasma citrulline concentrations in dogs treated with a multi-strain probiotic, suggesting the restoration of the mucosal barrier. Thus, more studies are required in order to accurately evaluate the potential use of citrulline as a biomarker in CIE in dogs. Soluble receptor for advanced glycation end products The receptor of advanced glycation end products (RAGE) is a multi-ligand PRR (Heilmann et al., 2014), which is implicated in dogs with CIE (Heilmann and Allenspach, 2017). Signaling pathways of RAGE lead to the activation of several kinases, including the activation and nuclear translocation of NF-Kb. As a consequence of this activation, inflammatory cells are recruited and a proinflammatory microenvironment is installed. Soluble RAGE (sRAGE) is a truncated variant of RAGE. sRAGE functions as an anti-inflammatory decoy receptor that can sequester RAGE ligands, preventing their interaction with cell surface RAGE. Thus, sRAGE has the capacity to modulate and abolish cell signaling, nullifying the proinflammatory effect of ligands for this receptor (Heilmann and Allenspach, 2017; Heilmann and Steiner, 2018).

Dogs with CIE have significantly decreased serum sRAGE concentrations (Heilmann and Steiner, 2018), as specifically demonstrated in dogs with IBD (Heilmann et al., 2014). However, in this study, researchers could not determine whether this decrease in sRAGE concentrations was due to its consumption or decreased production. The lower concentrations of 
circulating sRAGE, functioning as a decoy receptor, might permit the ligand-RAGE binding, leading to the activation of RAGE pathways and consequently, potentiating the inflammatory response. In addition, SRAGE concentrations were not correlated with CIBDAI or histopathologic disease scores. However, only esophagogastroduodenoscopies were carried out and lesions in the ileum or colon could have been unintentionally disregarded, which could be considered as a limitation of this study (Heilmann et al., 2014).

Heilmann and Steiner (2018) have reported the potential of serum sRAGE concentration to assess the response to treatment in dogs with CIE, since serum sRAGE concentrations stabilized in dogs only after achieving complete clinical remission (Heilmann et al., 2014).

Assuming that sRAGE has the capacity to downregulate the proinflammatory response mediated by RAGE, a possible therapeutic strategy for dogs with CIE using this anti-inflammatory receptor should be further investigated (Heilmann et al., 2014).

Metabolite profile. Oxidative stress is hypothesized to play a role in the pathogenesis of IBD, resulting from a significant disproportion between the production of reactive oxygen species (ROS) and their elimination by antioxidants (Rubio et al., 2017).

Rubio et al. (2017) have analyzed a profile of several serum biomarkers of oxidative stress in dogs with idiopathic IBD and compared it to healthy dogs. In order to determine the antioxidant response, biomarkers of total antioxidant status, such as Trolox equivalent antioxidant capacity (TEAC), cupricreducing antioxidant capacity (CUPRAC), and ferricreducing ability of the plasma (FRAP), were evaluated. In addition, individual antioxidant biomarkers were analyzed, including total thiol concentrations and paraoxonase 1 (PON1) activity. Moreover, biomarkers of oxidant status, such as ferrous oxidation-xylenol orange (FOX), thiobarbituric acid reactive substances (TBARS), and ROS production concentrations were measured in order to evaluate oxidative damage. Results showed a significant reduction in TEAC, CUPRAC, thiol, and PON1 in dogs with IBD, in comparison with healthy dogs, revealing a decrease in the antioxidant response. Interestingly, no alteration was detected in serum FRAP. This finding might be explained as FRAP, which may vary according to its individual antioxidants contributors, unlike the other total antioxidant capacity assays. Hence, the use of different methods to determine the total antioxidant status is recommended. Moreover, TBARS, FOX, and ROS levels were increased, suggesting the presence of an elevated oxidative stress status in canine IBD. The authors referred that this intense and permanent oxidative stress could lead to the decrease in antioxidant resources, surpassing the body production capacity. Therefore, the decreased antioxidant response observed could be justified. In addition, the authors suggested that the lymphocytes and plasma cells present in the inflamed intestinal mucosa of dogs with IBD might be a source of systemic ROS production (Rubio et al., 2017).

Future studies should be carried out in order to assess the potential of this metabolite profile in evaluating the oxidative stress response in dogs with IBD.

\section{Fecal and urinary biomarkers}

Alpha 1-proteinase inhibitor. Alpha 1-proteinase inhibitor $(\alpha 1 \mathrm{PI})$ is a major proteinase inhibitor that is synthesized in the liver (Heilmann and Steiner, 2018), macrophages, and in the intestinal mucosa (Wdowiak et al., 2013). This plasma protein has a similar weight to albumin and both can be lost from the interstitium to the GI lumen at the same rate in PLE. However, unlike albumin, $\alpha 1$ PI is not affected by proteolysis (Heilmann and Steiner, 2018), and it is able to remain unaltered throughout the intestinal tract, allowing its extraction and measurement from fecal samples (Cerquetella et al., 2010; Wdowiak et al., 2013; Heilmann and Steiner, 2018). Elevated fecal canine $\alpha 1$ PI concentrations are clinically useful as a marker of GI protein loss, and at the same time it is a risk factor for negative outcomes in CIE (Berghoff and Steiner, 2011).

This biomarker has shown to be correlated with histopathologic lesions seen in dogs with PLE, such as lacteal dilatation and/or crypt abscesses (Heilmann et al., 2016). Similarly, Murphy et al. (2003) observed higher concentrations of fecal $\alpha 1 \mathrm{PI}$ in dogs with GI disorders showing histologic abnormalities, such as IBD and lymphangiectasia. Furthermore, the authors did not correlate fecal $\alpha 1 \mathrm{PI}$ concentrations with serum albumin concentrations. This finding could be explained based on the assumption that hypoalbuminemia would only develop if protein loss was severe enough to exceed hepatic production capacity. Hence, the assessment of fecal $\alpha 1$ PI concentrations may identify patients who have ongoing intestinal protein loss, before the occurrence of clinical signs (Berghoff and Steiner, 2011), making this biomarker principally useful in detecting protein loss during early stages of the disease (Murphy et al., 2003). Furthermore, it might also be useful to differentiate hepatic causes from GI protein loss (Heilmann and Steiner, 2018).

Fecal $\alpha 1$ PI concentrations may vary significantly from one day to another. Ideally, fecal samples should be collected on 3 consecutive days and a 3-day mean should be taken in order to improve test accuracy (Allenspach, 2015; Heilmann and Steiner, 2018). Despite its high sensitivity, the measurement of fecal a1PI is not considered a specific biomarker for this condition, as GI protein loss can be associated with several other GI systemic disorders; however, it may have a role in monitoring disease progression and treatment response (Collins, 2013).

Immunoglobulin A. In mucosal lymphoid tissues, IgA is largely produced. This antibody (Maeda et al., 2013) is secreted in the intestinal mucosa mainly in its dimeric form (Allenspach, 2011) and plays a crucial role in maintaining intestinal homeostasis (Maeda et al., 
2013). IgA provides a first line of immune protection at mucosal surfaces (Maeda et al., 2013), by preventing commensal and dietary antigens from triggering an immune response (Lee et al., 2015). The interaction between commensals and cells of the intestinal immune system is critical to the secretion of IgA, as it promotes B lymphocytes to switch its class to IgAproducing plasma cells (Lee et al., 2015) via T celldependent and independent mechanisms (Eissa et al., 2019). Even though IgA may be detected in different biological samples, this biomarker will be addressed in this section, since its fecal concentrations reflect more accurately the inflammation degree, in comparison with serum concentrations (Maeda et al., 2013; Heilmann and Steiner, 2018).

German shepherd dogs are known for their predisposition to polymorphisms in the gene encoding TLR-5. In addition, this breed is known for its abnormal IgA production (Maeda et al., 2013; Lee et al., 2015), being highly susceptible to CIE (Jergens and Simpson, 2012). However, Lee et al. (2015) failed to correlate IgA-positive plasma cells in the mucosa of dogs with CIE with single nucleotide polymorphisms in the gene encoding TLR-5.

The involvement of IgA in the pathogenesis mechanism remains unclear (Maeda et al., 2013); however, it has been considered whether an impaired function of IgA-producing plasma cells and decreased fecal IgA concentrations could be a cause or a consequence of the disease (Heilmann and Steiner, 2018). Studies have observed decreased concentrations of $\operatorname{IgA}$ in the duodenum and feces of dogs with IBD, without observing any change in serum $\operatorname{IgA}$ concentrations (Maeda et al., 2013). In addition, researchers failed to correlate fecal IgA concentration with clinical severity. Despite detecting decreased fecal IgA concentrations in dogs with CIE, available data are inconclusive about its clinical utility as a biomarker (Heilmann and Steiner, 2018).

Bromotyrosine. Eosinophils are typically present in low numbers in the intestinal mucosa of healthy dogs. Eosinophilic enteritis, the second most commonly diagnosed form of IBD in dogs, is characterized by a mixed infiltration of inflammatory cells, predominantly eosinophils. During inflammatory processes, eosinophils are activated and migrate to the villi, where degranulation occurs. Eosinophils release various highly cytotoxic granule proteins, such as eosinophil peroxidase, eosinophil cationic protein, eosinophilderived neurotoxin, and major basic protein, resulting in tissue damage and dysfunction (Bastan et al., 2017). Bromotyrosine (3-BrY) is the stable metabolite of eosinophil peroxidase, a potent granular cytotoxic hemeprotein (Sattasathuchana et al., 2015). 3-BrY concentrations reflect eosinophilic inflammation, according to the eosinophilic component that can be present in the cellular infiltrate of the lamina propria (Heilmann and Steiner, 2018). This biomarker can be detected in different biological samples. Nevertheless, the focus of this analysis is on feces, as reports have evidenced that the assessment of 3-BrY concentrations in fecal samples reflect the level of eosinophil activation in the GI tract more accurately than serum biomarkers (Sattasathuchana et al., 2019).

A recent study revealed higher concentrations of fecal 3-BrY concentrations in dogs with CIE; however, sensitivity and specificity to differentiate between the different forms of CIE are yet to be determined (Sattasathuchana et al., 2019). Sattasathuchana et al. $(2015,2017)$ assessed 3-BrY concentrations in the serum of dogs with CIE. Researchers showed increased serum 3-BrY concentrations in dogs with eosinophilic gastroenteritis, as well as in dogs with lymphocyticplasmacytic enteritis (Sattasathuchana et al., 2015). These findings highlight the pathophysiological role of eosinophil activation in dogs with CIE that have these types of cellular infiltrates. Later, the same authors observed increased serum 3-BrY concentrations in dogs with FRE and IRE in comparison with healthy dogs. Additionally, higher concentrations were noted in IRE dogs, suggesting an increased severity of inflammation in dogs with this form of CIE. No significant correlation was established with clinical severity, as determined by CCECAI (Sattasathuchana et al., 2017). Researchers proposed that the simultaneous use of these two independent predictors might improve diagnosis specificity and prediction of the most likely outcome.

The clinical utility of $3-\mathrm{BrY}$ should be further investigated before the use of this biomarker can be recommended for clinical practice (Heilmann and Steiner, 2018).

$N$-methylhistamine. In canine CIE, mast cells participate in inflammatory processes through the release of multiple inflammatory mediators in the intestinal mucosa, such as histamine (Wdowiak et al., 2013). Serum histamine concentrations may directly reflect the degree of mast cell activation; however, it is not usually measured due to its short half-life (Anfinsen et al., 2014). A stable metabolite of histamine, N-methylhistamine (NMH) has been suggested as a proinflammatory marker of mast cell degranulation and GI inflammation (Berghoff and Steiner, 2011; Heilmann and Steiner, 2018), as it can more accurately reflect the overall mast cell activity (Anfinsen et al., 2014). NMH is generated via the histamine $\mathrm{N}$-methyltransferase enzyme system (Berghoff et al., 2014) and can be readily measured from urine and fecal specimens (Anfinsen et al., 2014; Berghoff et al., 2014).

Studies failed to establish a correlation between fecal and urinary NMH concentrations and clinical severity, as well as with the degree of mast cell infiltration (Anfinsen et al., 2014; Berghoff et al., 2014). However, higher quantities of intestinal mast cells were observed in dogs responding to dietary trials, when compared to those requiring immunosuppressive treatment, suggesting that mast cell activation might 
be implicated in the pathogenesis of FRE (Anfinsen et al., 2014). Unlike the previous study, Berghoff et al. (2014) observed a significant correlation between urinary $\mathrm{NMH}$ concentrations and the histological grade of inflammation, implying that urinary $\mathrm{NMH}$ concentrations might be a more accurate indicator of disease severity when compared to fecal concentrations. $\mathrm{NMH}$ has been reported as a potentially useful biomarker in clinical practice; however, more studies are needed in order to determine its sensitivity and specificity (Heilmann and Steiner, 2018).

Leukotriene E4. Leukotriene E4 (LTE4) is a proinflammatory product derived from 5-lipoxygenase that contributes to the inflammatory response by increasing vessel permeability, chemotaxis, and mucous secretion in the colonic mucosa (Im Hof et al., 2012; Wdowiak et al., 2013; Dumusc et al., 2014). This metabolite of the cysteinyl leukotriene enzymatic pathway is obtainable in urine samples. The collection of multiple samples in short periods is recommended in order to more accurately estimate the LTE4 production (Im Hof et al., 2012).

Researchers showed no significant difference in urinary LTE4 concentrations in dogs with IBD, compared to dogs diagnosed with FRE. Yet, both groups presented significantly higher concentrations in comparison with healthy dogs. These findings might indicate how important the contribution of cysteinyl leukotriene pathway activation can be to the intestinal inflammation. Hence, the potential use of 5-lipoxygenase inhibitors or leukotriene receptor antagonists for therapeutic interventions should be further investigated. Moreover, LTE4 concentrations were not correlated with clinical severity, as determined by CIBDAI (Im Hof et al., 2012).

The levels of LTE4 may have potential to be used as biomarkers in canine CIE. Yet, future studies with a larger number of dogs are required in order to support the existing data (Im Hof et al., 2012).

Calprotectin. Calprotectin, also referred as S100A8/ A9, is a heterodimeric protein complex (Collins, 2013) that belongs to the S100/calgranulin family of damageassociated molecular pattern (DAMP) molecules (Heilmann et al., 2018; Heilmann and Steiner, 2018). Activated macrophages and neutrophils express and release calprotectin in the extracellular space (Heilmann and Steiner, 2018). However, this calcium and zinc-binding protein (Celi et al., 2019) can also be induced in epithelial cells (Heilmann and Steiner, 2018). Calprotectin is a ligand for TLR-4 (Heilmann et al., 2019), which is upregulated in dogs with idiopathic IBD, as reported by Heilmann et al. (2012). Thus, it is speculated that calprotectin might be involved in the expression of proinflammatory cytokines and chemokines (Heilmann and Steiner, 2018), playing a role in acute and chronic inflammation (Heilmann et al., 2019). Even though calprotectin can be measured in different biological samples, it will be addressed in this section since fecal concentrations of this biomarker are reported as more specific for detecting gastrointestinal inflammatory processes (Heilmann and Steiner, 2018). Calprotectin is considered stable in natural feces, thus allowing a sample collection in the dog's home environment (Otoni et al., 2018). One study revealed increased concentrations of fecal calprotectin in dogs with IBD, at the time of diagnosis, which decreased significantly after treatment (Otoni et al., 2018). Similarly, Grellet et al. (2013) observed higher fecal calprotectin concentrations in dogs with chronic diarrhea. Furthermore, fecal calprotectin concentrations have been positively correlated with clinical and histopathologic severity (Grellet et al., 2013; Heilmann et al., 2018; Otoni et al., 2018). When evaluating the histopathologic lesions, researchers noted a correlation between fecal calprotectin concentration and lymphocytes in the ileal lamina propria, rather than a correlation with intestinal lamina propria neutrophils and macrophages. This finding could be explained, as calprotectin expression might reflect the activity of these cells, instead of their quantity (Heilmann et al., 2018). Moreover, this biomarker appears to have potential in differentiating the various forms of CIE, with dogs responsive to immunosuppressive treatment and showing higher concentrations (Heilmann et al., 2018). However, assessing fecal calprotectin in combination with serum CRP and CCECAI score was demonstrated to increase the ability to differentiate between these conditions (Heilmann et al., 2018). In addition, this biomarker seems to be clinically useful in predicting the response to treatment in dogs with CIE (Heilmann and Steiner, 2018), as it is specific for the GI tract (Heilmann et al., 2018). Furthermore, the authors also verified that fecal calprotectin concentrations did not correlate with serum CRP concentrations, suggesting that the intestinal inflammation in dogs with CIE is not related to the systemic inflammatory response (Heilmann et al., 2018). This biomarker appears to be useful for the noninvasive evaluation of intestinal inflammation because of its potential to monitor disease intensity and detect both active and inactive periods of the disease (Otoni et al., 2018).

Calprotectin can also be detected in the serum. Calprotectin concentrations are reported to be increased in the serum of dogs with CIE; however, it is not specific to the GI tract (Heilmann and Steiner, 2018). Researchers documented an increase in serum calprotectin concentrations in dogs with idiopathic IBD, compared with healthy dogs (Heilmann et al., 2012). Contrarily, Otoni et al. (2018) showed no differences in serum calprotectin concentrations between healthy dogs and dogs with idiopathic IBD or in dogs before and after treatment. Additionally, both studies failed to correlate serum calprotectin concentrations with CIBDAI scores, as well as with histopathologic severity. Serum calprotectin appears to be useful in detecting inflammation; however, the 
inability to identify the exact inflamed organ limits its clinical utility (Heilmann et al., 2012).

Calprotectin has been considered a biomarker with a great potential to be used in dogs with CIE, particularly in monitoring GI inflammation (Heilmann et al., 2018; Celi et al., 2019).

Calgranulin C. Calgranulin C, also referred as $\mathrm{S} 100 \mathrm{~A} 12$, is an endogenous DAMP involved in phagocyte activation (Heilmann et al., 2014). This calcium-binding protein (Wdowiak et al., 2013) is principally expressed and secreted by activated neutrophils, macrophages, and monocytes (Hanifeh et $a l ., 2018)$ and has a significant role in inflammatory immune responses (Heilmann and Steiner, 2018). After being released in the extracellular space (Hanifeh et al., 2018), it works as a ligand for RAGE (Heilmann et al., 2016; Heilmann and Steiner, 2018). Binding to this PRR can trigger signaling pathways that lead to the activation of NF-kb, resulting in the production of proinflammatory cytokines and chemokines. Also, a positive feedback on the expression of transmembrane RAGE itself leads to the perpetuation and amplification of the inflammatory response and consequently to tissue damage (Heilmann et al., 2014; Hanifeh et al., 2018). Calgranulin $\mathrm{C}$ is quite a sensitive and specific biomarker for localized inflammatory disorders, such as GI inflammation. This biomarker might be detected in different biological samples. However, concentrations in serum might also be increased in other inflammatory disorders. Thus, this biomarker will be addressed in this section, since its fecal concentrations are more specific for detecting gastrointestinal inflammatory processes (Heilmann and Steiner, 2018).

Calgranulin $\mathrm{C}$ can be detected in fecal samples. Higher concentrations of fecal calgranulin $\mathrm{C}$ have been detected in dogs with IBD (Heilmann et al., 2014), as well as in dogs with CIE, in general (Hanifeh et al., 2018). This finding indicates an increased infiltration of phagocytes, supporting the idea that phagocyte activation plays a role in the pathogenesis of the disease (Heilmann et al., 2014). Furthermore, studies have demonstrated a significant correlation between fecal calgranulin $\mathrm{C}$ concentrations and clinical severity (Heilmann, et al., 2014), endoscopic lesions (Heilmann et al., 2014, 2018), as well as histopathologic alterations in the colon, but not with the severity of histopathologic lesions overall (Heilmann et al., 2014). Additionally, fecal calgranulin $\mathrm{C}$ concentrations have been correlated with a negative outcome (Hanifeh et al., 2018). Moreover, researchers showed the potential of fecal calgranulin $\mathrm{C}$ concentrations in distinguishing dogs with CIE that are more likely to respond to dietary trials, antibiotic treatment, or immunosuppressive therapy (Heilmann et al., 2016). Results also indicated the utility of this biomarker in predicting the lack of response to treatment in dogs with CIE, suggesting its prognostic value.
A recent study reported increased levels of calgranulin $\mathrm{C}$ in the intestinal mucosa of dogs with CIE (Hanifeh et al., 2018). The authors established a significant correlation between colonic mucosal calgranulin $\mathrm{C}$ concentrations and the severity of epithelial injury. Furthermore, they also associated increased mucosal calgranulin $\mathrm{C}$ concentrations with the presence of macrophages or neutrophil inflammatory infiltrate components.

Calprotectin has been considered as a highly attractive biomarker to be used in canine CIE (Heilmann et al., 2018). The measurement of calgranulin $\mathrm{C}$ in fecal samples, as a non-invasive test, is reported to be particularly advantageous for monitoring GI inflammation (Celi et al., 2019).

\section{Intestinal alkaline phosphatase}

Intestinal alkaline phosphatase (IAP) is an isoenzyme of alkaline phosphatase that contributes positively to the maintenance of homeostatic conditions of the intestinal flora (Ide et al., 2016). It is expressed in high quantities in the duodenum, mainly in villus enterocytes, having a gradual decline throughout the rest of the intestinal tract (Celi et al., 2019). During digestion IAP is not dissolved, thus fecal concentrations reflect the original expression in epithelial cells (Ide et al., 2016). This biomarker has been reported as an indicator of mature enterocytes (Celi et al., 2019).

Important functions carried out by IAP include $\mathrm{pH}$ modulation, assimilation of organophosphorus acid, and fat absorption into the intestinal tract. In the intestinal mucosa, IAP has an important role of protection, as it is capable of lipopolysaccharide dephosphorylation. This is a component of the outer cell membrane of Gram-negative bacteria, which are overrepresented in dogs with CIE. In this way, dogs with CIE simultaneously have a significant number of intestinal lipopolysaccharides and a defective capacity to neutralize them. IAP neutralizes bacteria endotoxic properties and protects the intestinal mucosa from the detrimental effects of endotoxins; abnormalities in both IAP expression and function may alter the lipopolysaccharide, and result in endotoxin-induced inflammation or in an abnormal response against the intestinal flora (Ide et al., 2016).

A significant decrease in IAP's expression has been documented in the duodenal mucosa of dogs with CIE, particularly in those with moderate and severe disease (Ide et al., 2016). Researchers hypothesized that a decrease in IAP production might be either a cause or a consequence of the intestinal inflammation by increasing the intestinal mucosa exposure to active endotoxins. Additionally, researchers noted an increased expression of IAP in the duodenum, compared with the colon in affected dogs (Ide et al., 2016).

Future studies need to be carried out in order to investigate the role of IAP in canine CIE pathogenesis (Ide et al., 2016), as well as to assess its potential as a biomarker (Heilmann and Steiner, 2018). 


\section{Intestinal microbiota}

The GI microbiota is a complex population of living microorganisms comprising bacteria, archaea, fungi, protozoa, and viruses (Honneffer et al., 2014; Redfern et al., 2017; Eissa et al., 2019). This highly complex ecosystem plays an essential role in GI health (Honneffer et al., 2014; Omori et al., 2017), mainly in digestion, absorption, energy metabolism, immune system development, and in the prevention of infections (Celi et al., 2019). A significant proportion of this system is bacterial species comprised in the phyla Bacteroidetes, Firmicutes, Proteobacteria, and Actinobacteria.

Intestinal microbiota and the host immune system have a complex mutual relationship (Eissa et al., 2019). In a balanced environment, resident microbiota compete against pathogens for available resources and space in the GI tract, preventing pathogen colonization (Omori et al., 2017). In addition, commensals produce shortchain fatty acids (SCFAs) through the fermentation of substrates, such as nondigested dietary residues, endogenous mucus, and sloughed epithelial cells (Xu et al., 2016; Eissa et al., 2019). As a result, commensals obtain energy for their metabolism and allow epithelial cell growth (Omori et al., 2017). In this mutualistic interaction, the host contributes the nutrients and niches that are crucial for microbiota colonization (Eissa et al., 2019).

Gut microbiota also contribute to the homeostasis of systemic immunity (Omori et al., 2017) by promoting self-tolerance (Redfern et al., 2017). Hence, an imbalance in bacteria populations within the GI tract, defined as dysbiosis, can significantly affect their functions (Redfern et al., 2017). Alterations of intestinal microbiota have been associated with CIE, thus an inappropriate activation of immune responses against GI microbiota is thought to contribute to the mechanisms of the disease (Omori et al., 2017).

Dogs with CIE have been associated with a lower microbiota diversity (Eissa et al., 2019), characterized by an overrepresentation of the phylum Proteobacteria (Honneffer et al., 2014), particularly in the Enterobacteriaceae family (Simpson and Jergens, 2011) and in the Delftia genus (Kalenyak et al., 2018). Increases in the phylum Actinobacteria were also reported (Honneffer et al., 2014), particularly in the genus Corynebacterium (Kalenyak et al., 2018).

Affected dogs have been characterized by a decrease in the phylum Firmicutes (Honneffer et al., 2014), specifically in the Clostridiales order (Eissa et al., 2019). With regard to this phylum, Xu et al. (2016) observed that dogs demonstrating higher CCECAI scores showed a gradual decrease in Lactobacillus strains. These commensal organisms carry out important functions, such as the production of SCFAs (Redfern et al., 2017) and the downregulation of pro-inflammatory cytokines, as demonstrated in murine models (Xu et al., 2016). Interestingly, Kalenyak et al. (2018) showed there was a decrease in the Enterococcus genus from the same phylum.

Furthermore, lower populations of the phylum Bacteroidetes were shown (Honneffer et al., 2014), particularly in the Bacteroidales order, as reported by Eissa et al. (2019). Yet, Kalenyak et al. (2018) observed increased populations of the Bacteroides genus in dogs with FRE and IBD after treatment. Bacteroides are considered valuable for their ability to reduce carbohydrates and breakdown of bile acid. Therefore, the potential use of these strains as a marker to assess response to treatment has been suggested.

Discrepancies among studies analyzing microbiota composition could be justified by the different sampling methods to evaluate microbiota, differences in study population, diet variability within the individuals (Kalenyak et al., 2018), the use of medications, such as antibiotics, and the different washout periods applied (Omori et al., 2017).

Recently, researchers developed a mathematical algorithm to evaluate alterations in the intestinal microbiota in fecal samples (AlShawaqfeh et al., 2017). The fecal dysbiosis index (DI) consists of a quantitative polymerase chain reaction panel to assess eight bacterial groups that are normally changed in dogs with CIE, including Blautia, Clostridium hiranonis, Escherichia coli, Faecalibacterium, Fusobacterium, Streptococcus, Turicibacter, and total bacteria. This tool evaluates the occurrence of dysbiosis and may also be useful to track whether the microbiota normalizes in response to treatment.

In order to estimate the clinical utility of DI as a tool to analyze microbiota dysbiosis in dogs with CIE and their response to treatment, future studies have to be carried out (AlShawaqfeh et al., 2017).

\section{Final considerations}

Canine CIE comprehend a group of idiopathic GI disorders with a chronic cyclical remission-relapse nature, which are considered mediated immunologically (Jergens and Simpson, 2012; Heilmann and Steiner, 2018). This condition has been recognized, so far, as the biggest cause for chronic GI signs in dogs. However, it is currently overdiagnosed in clinical practice, possibly due to the difficulties inherent to the diagnosis process (Somu et al., 2017). This evidence, associated to the fact that their etiologies and pathogenesis mechanisms remains partially unclear, emphasizes the need for further investigation on this subject (Wdowiak et al., 2013).

Currently, the diagnosis and monitoring of CIE rely predominantly on clinical, laboratory, endoscopic, and histologic parameters (Cerquetella et al., 2010; Wdowiak et al., 2013; Allenspach, 2015; Moser et al., 2018). However, these methods have several limitations. In addition to the subjective nature of clinical score systems, the clinical signs usually do not reflect intestinal inflammation (Heilmann et al., 2018). Furthermore, endoscopic and histopathologic 
procedures are semi-invasive, expensive, and unlikely to be frequently carried out (Heilmann et al., 2014). Additionally, the interpretation of histopathologic tissue specimens depends significantly on interobserver variability. In summary, all these limitations constitute a big challenge to the approach and management of patients with CIE. To provide a solution for all of these concerns, biomarkers appear as a more objective and non-invasive tool that can have great advantages in estimating diagnosis, defining disease severity, and predicting the most likely outcome (Wdowiak et al., 2013; Otoni et al., 2018).

Biomarkers have a great potential in helping in the diagnosis and management of dogs with CIE. Based on the literature used for this study, one may argue that fecal calprotectin and fecal calgranulin $\mathrm{C}$ are promising biomarkers of intestinal inflammation (Celi et al., 2019). When compared to other biomarkers, the concentrations of these two DAMP molecules have been positively correlated with clinical and histopathological severity (Grellet et al., 2013; Heilmann et al., 2014, 2018; Otoni et al., 2018). Furthermore, their usefulness in predicting clinical outcomes (Hanifeh et al., 2018; Heilmann and Steiner, 2018), as well as in differentiating the various forms of CIE, has been proven (Heilmann et al., 2016, 2018). Moreover, calprotectin and calgranulin $C$ have been analyzed the most through clinical trials and articles substantiating its value more than the other biomarkers. Even though the remaining biomarkers discussed in this article have not presented results as solid as calprotectin and calgranulin $\mathrm{C}$, future investigations should be carried out with the goal of contributing to the current knowledge advancement of biomarkers' clinical utility in canine CIE. It is also important to flag that, even though the investigations in the intestinal microbiome are recent, it is a promising area with great potential to be used in clinical practice. In addition, a single biomarker cannot assuredly predict disease severity, progression, response to treatment and clinical outcome. Therefore, in order to achieve greater accuracy, it would be beneficial if these tools are used in conjunction with the contemporary ones (Collins, 2013).

In the present research, it is important to point out its limitations. Firstly, when reporting clinical trials, the different methods for the detection of biomarkers expression, as well as their sensitivity, specificity, and cut-off values were not taken into consideration. This limitation can be justified, since the majority of the studies analyzed in this article did not have this information available. Moreover, there are not enough clinical trials behind some of the reported biomarkers that could support the conclusion about their clinical utility. In order to address these limitations, it is of utmost urgency to raise awareness about biomarkers' usefulness in this field. Furthermore, although recent investigations on biomarkers' utility in dogs with CIE have been carried out, none of them are routinely used in clinical practice (Heilmann et al., 2018). Hence, one can draw the conclusion that future research is needed in order to better determine the usefulness of these tools in diagnosing and managing CIE in dogs.

\section{Conflict of interest}

The authors declare that there is no conflict of interest.

\section{References}

Abraham, C. and Cho, J.H. 2009. Mechanisms of disease Inflammatory Bowel Disease. N. Engl. J. Med. 361(21), 2066-2078.

Allenspach, K. 2011. Clinical immunology and immunopathology of the canine and feline intestine. Vet. Clin. Small. Anim. 41(2), 345-360.

Allenspach, K. 2015. Diagnosis of small intestinal disorders in dogs and cats. Clin. Lab. Med. 35(3), 521-534.

Allenspach, K., Culverwell, C. and Chan, D. 2016. Long-term outcome in dogs with chronic enteropathies: 203 cases. Vet. Rec. 178(15), 362368.

Allenspach, K., Mochel, J.P., Du, Y., Priestnall, S.L., Moore, F., Slayter, M. Rodrigues, A., Ackermann, M., Krockenberger M., Mansell, J., WSAVA GI Standardization Working Group; Luckschander, L., Wang, C., Suchodolski, J., Berghoff, N. and Jergens, A.E. 2018. Correlating gastrointestinal histopathologic changes to clinical disease activity in dogs with idiopathic inflammatory bowel disease. Vet. Pathol. 56(3), 435-443.

Allenspach, K., Wieland, B., Grone, A. and Gaschen, F. 2007. Chronic enteropathies in dogs: evaluation of risk factors for negative outcome. J. Vet. Intern. Med. 21, 700-708.

AlShawaqfeh, M., Wajid, B., Minamoto, Y., Markel, M., Lidbury, J., Steiner, J., Serpedin, E. and Suchodolski, J.S. 2017. A dysbiosis index to assess microbial changes in fecal samples of dogs with chronic inflammatory enteropathy. FEMS Microbiol. Ecol. 93(11), 1-8.

Anfinsen, K.P., Berghoff, N., Priestnall, S.L., Suchodolski, J.S., Steiner, J.M. and Allenspach, K. 2014. Urinary and faecal N-methylhistamine concentrations do not serve as markers for mast cell activation or clinical disease activity in dogs with chronic enteropathies. Acta Vet. Scand. 56(1), 90. https://doi.org/10.1186/s13028-014-0090-y

Aono, K., Azuma, Y.T., Nabetani, T., Hatoya, S., Furuya, M., Miki, M., Hirota, K., Fujimoto, Y., Nishiyama, K., Ogata, Y., Mochizuki, T. and Tani, H. 2019. Correlation between toll-like receptor 4 and nucleotide-binding oligomerization domain 2 (NOD2) and pathological severity in dogs with chronic gastrointestinal diseases. Vet. Immunol. Immunopathol. 210, 15-22.

Bastan, I., Robinson, N.A., Ge, X.N., Rendahl, A.K., Rao, S.P., Washabau, R.J. and Sriramarao, P. 2017. Assessment of eosinophil peroxidase as a potential 
diagnostic and prognostic marker in dogs with inflammatory bowel disease. Am. J. Vet. Res. 78(1), 36-41.

Berghoff, N., Hill, S., Parnell, N.K., Mansell, J., Suchodolski, J.S. and Steiner, J.M. 2014. Fecal and urinary N-methylhistamine concentrations in dogs with chronic gastrointestinal disease. Vet. J. 201(3), 289-294.

Berghoff, N. and Steiner, J.M. 2011. Laboratory Tests for the Diagnosis and Management of Chronic Canine and Feline Enteropathies. Vet. Clin. Small. Anim. 41(2), 311-328.

Celi, P., Verlhac, V., Pérez, C.E., Schmeisser, J. and Kluenter, A.M. 2019. Biomarkers of gastrointestinal functionality in animal nutrition and health. Anim. Feed Sci. 250, 9-31.

Cerquetella, M., Spaterna, A., Laus, F., Tesei, B., Rossi, G., Antonelli, E., Villanacci, V. and Bassotti, G. 2010. Inflammatory bowel disease in the dog: Differences and similarities with humans. World J. Gastroenterol. 16(9), 1050-1056.

Collins, M.T. 2013. Canine Inflammatory Bowel Disease: Current and Prospective Biomarkers for Diagnosis and Management. Vetlearn. 38(3), 1-7.

Dandrieux, J.R.S. 2016. Inflammatory bowel disease versus chronic enteropathy in dogs: are they one and the same? J. Small Anim. Pract. 57(11), 589599.

Day, M.J., Bilzer, T., Mansell, J., Wilcock, B., Hall, E.J., Jergens, A., Minami, T., Willard, M., Washabau, R. and WSAVA GI Standardization Working Group. 2008. Histopathological standards for the diagnosis of gastrointestinal inflammation in endoscopic biopsy samples from the dog and cat: a report from the World Small Animal Veterinary Association Gastrointestinal Standardization Group. J. Comp. Pathol. 138, 1-43.

Dumusc, S.D., Ontsouka, E.C., Schnyder, M., Hartnack, S., Albrecht, C., Bruckmaier, R.M. and Burgener, I.A. 2014. Cyclooxygenase-2 and 5-lipoxygenase in dogs with chronic enteropathies. J. Vet. Intern. Med. 28(6), 1684-1691.

Eissa, N., Kittana, H., Gomes-Neto, J.C. and Hussein, H. 2019. Mucosal immunity and gut microbiota in dogs with chronic enteropathy. Res. Vet. Sci. 122, $156-164$.

European Commission Health Research Directorate (ECHRD). 2010. Biomarkers for patient stratification. Brussels. Available via https:// ec.europa.eu/research/health/pdf/biomarkers-forpatient-stratification_en.pdf.

German, A.J., Day, M.J., Ruaux, C.G., Steiner, J.M., Williams, D.A. and Hall, E.J. 2003. Comparison of Direct and Indirect Tests for Small Intestinal Bacterial Overgrowth and Antibiotic Responsive Diarrhea in Dogs. J. Vet. Intern. Med. 17(1), 33-43.

Gerou-Ferriani, M., Allen, R., Noble, P.J.M., German, A.J., Caldin, M. and Batchelor, D.J. 2018.
Determining optimal therapy of dogs with chronic enteropathy by measurement of serum citrulline. J. Vet. Intern. Med. 32(3), 993-998.

Gram, W.D., Milner, R.J. and Lobetti, R. 2018. Chronic disease management for small animals. Can. Vet. J. 59(5), 499.

Grellet, A., Heilmann, R.M., Lecoindre, P., Feugier, A., Day, M.J., Peeters, D., Freiche, V., Hernandez, J., Grandjean, J., Suchodolski, J.S. and Steiner, J.M. 2013. Fecal calprotectin concentrations in adult dogs with chronic diarrhea. Am. J. Vet. Res. 74(5), 706711 .

Hall, E.J. 2009. Inflammatory bowel disease in dogs and cats. Hill's Pet Nutrition, Inc. Available via https:// protrain.hs.llnwd.net/e1/sitefiles/642/Documents/ GI\%20technical\%20booklet.pdf.

Hanifeh, M., Sankari, S., Rajamäki, M.M., Syrjä, P., Kilpinen, S., Suchodolski, J.M., Heilmann, R.M., Guadiano, P., Lidbury, J., Steiner, J.M. and Spillmann, T. 2018. S100A12 concentrations and myeloperoxidase activities are increased in the intestinal mucosa of dogs with chronic enteropathies. BMC Vet. Res. 14(1), 1-13.

Heilmann, R.M. and Allenspach, K. 2017. Patternrecognition receptors: signaling pathways and dysregulation in canine chronic enteropathiesbrief review. J. Vet. Diagn. Invest. 29(6), 781-787.

Heilmann, R.M., Berghoff, N., Mansell, J., Grützner, N., Parnell, N.K., Gurtner, C., Suchodolski, J.S. and Steiner, J.M. 2018. Association of fecal calprotectin concentrations with disease severity, response to treatment, and other biomarkers in dogs with chronic inflammatory enteropathies. J. Vet. Intern. Med. 32(2), 679-692.

Heilmann, R.M., Grellet, A., Allenspach, K., Lecoindre, P., Day, M.J., Priestnal, S.L., Toresson, L., Procoli, F., Grutzner, N., Suchodolski, J.S. and Steiner, J.M. 2014. Association between fecal S100A12 concentration and histologic, endoscopic, and clinical disease severity in dogs with idiopathic inflammatory bowel disease. Vet. Immunol. Immunopathol. 158, 156-166.

Heilmann, R.M., Jergens, A., Ackermann, M.R., Barr, J.W., Suchodolski, S. and Steiner, J.M. 2012. Serum calprotectin concentrations in dogs with idiopathic inflammatory bowel disease. Am. J. Vet. Res. 73(12), 1900-1907.

Heilmann, R.M., Nestler, J., Schwarz, J., Grützner, N., Ambrus, A., Seeger, J., Suchodolski, J. S., Steiner, J.M. and Gurtner, C. 2019. Mucosal expression of S100A12 (calgranulin C) and S100A8/A9 (calprotectin) and correlation with serum and fecal concentrations in dogs with chronic inflammatory enteropathy. Vet. Immunol. Immunopathol. 211, 64-74.

Heilmann, R.M., Parnell, N.K., Grützner, N., Mansell, J., Berghoff, N., Schellenberg, S., Reusch, C.E., Suchodolski, J.S. and Steiner, J.M. 2016. Serum and 
fecal canine $\alpha 1$-proteinase inhibitor concentrations reflect the severity of intestinal crypt abscesses and/ or lacteal dilation in dogs. Vet. J. 207, 131-139.

Heilmann, R.M. and Steiner, J.M. 2018. Clinical utility of currently available biomarkers in inflammatory enteropathies of dogs. J. Vet. Intern. Med. 32(5), 1495-1508.

Heilmann, R.M. and Suchodolski, J.S. 2015. Is inflammatory bowel disease in dogs and cats associated with a Th1 or Th2 polarization? Vet. Immunol. Immunopathol. 168, 131-134.

Honneffer, J.B., Minamoto, Y. and Suchodolski, J.S. 2014. Microbiota alterations in acute and chronic gastrointestinal inflammation of cats and dogs. World J. Gastroenterol. 20(44), 16489-16497.

Ide, K., Kato, K., Sawa, Y., Hayashi, A., Takizawa, R. and Nishifuji, K. 2016. Comparison of the expression, activity, and fecal concentration of intestinal alkaline phosphatase between healthy dogs and dogs with chronic enteropathy. Am. J. Vet. Res. 77(7), 721729.

Im Hof, M., Schnyder, M., Hartnack, S., StankeLabesque, F., Luckschander, N. and Burgener, I.A. 2012. Urinary leukotriene E4 concentrations as a potential marker of inflammation in dogs with inflammatory bowel disease. J. Vet. Intern. Med. 26(12), 269-274.

Jergens, A.E., Crandell, J., Morrison, J.A., Deitz, K., Pressel, M., Ackermann, M., Suchodolski, J.S., Steiner, J.M. and Evans, R. 2010. Comparison of oral prednisone and prednisone combined with metronidazole for induction therapy of canine inflammatory bowel disease: A randomizedcontrolled trial. J. Vet. Intern. Med. 24(2), 269-277.

Jergens, A., Schreiner, C.A., Frank, D.E., Niyo, Y., Ahrens, F.E., Eckersall, P.D., Benson, T.J. and Evans, R. 2003. A Scoring Index for Disease Activity in Canine Inflammatory Bowel Disease. J. Vet. Intern. Med. 17(3), 291-297.

Jergens, A. and Simpson, K.W. 2012. Inflammatory bowel disease in veterinary medicine. Front. Biosci. 4(4), 1404-1419.

Jergens, A., Sonea, I.M., O’Connor, A.M., Kauffman, L.K., Grozdanic, S.D., Ackermann, M.R. and Evans, R.B. 2009. Intestinal cytokine mRNA expression in canine inflammatory bowel disease: A meta-analysis with critical appraisal. Comp. Med. 59(2), 153-162.

Junginger, J., Schwittlick, U., Lemensieck, F., Nolte, I. and Hewicker-Trautwein, M. 2012. Immunohistochemical investigation of Foxp3 expression in the intestine in healthy and diseased dogs. Vet. Res. 43(1), 23. https://doi. org/10.1186/1297-9716-43-23.

Kalenyak, K., Isaiah, A., Heilmann, R.M., Suchodolski, J.S. and Burgener, I.A. 2018. Comparison of the intestinal mucosal microbiota in dogs diagnosed with idiopathic inflammatory bowel disease and dogs with food-responsive diarrhea before and after treatment. FEMS Microbiol. Ecol. 94(2), 1-11.

Kołodziejska-Sawerska, A., Rychlik, A., Depta, A., Wdowiak, M., Nowicki, M. and Kander, M. 2013. Cytokines in canine inflammatory bowel disease. Pol. J. Vet. Sci. 16(1), 165-171.

Lee, A., Kathrani, A., Priestnall, S.L., Smith, K., Werling, D. and Allenspach, K. 2015. Lack of correlation between mucosal immunoglobulin A-positive plasma cell numbers and TLR5 genotypes in German shepherd dogs with idiopathic chronic enteropathy. J. Comp. Pathol. 152(2-3), 201-205.

Maeda, S., Ohno, K., Fujiwara-Igarashi, A., Uchida, K. and Tsujimoto, H. 2016. Changes in Foxp3-positive regulatory $\mathrm{T}$ cell number in the intestine of dogs with idiopathic inflammatory bowel disease and intestinal lymphoma. Vet. Pathol. 53(1), 102-112.

Maeda, S., Ohno, K., Nakamura, K., Uchida, K., Nakashima, K., Fukushima, K., Tsukamoto, A., Goto-Koshino, Y., Fujino, Y. and Tsujimoto, H. 2012. Mucosal imbalance of interleukin-1 $\beta$ and interleukin-1 receptor antagonist in canine inflammatory bowel disease. Vet. J. 194(1), 66-70.

Maeda, S., Ohno, K., Uchida, K., Nakashima, K., Fukushima, K., Tsukamoto, A., Nakajima, M., Fujino, Y. and Tsujimoto, H. 2013. Decreased immunoglobulin a concentrations in feces, duodenum, and peripheral blood mononuclear cells of dogs with inflammatory bowel disease. J. Vet. Intern. Med. 27(1), 47-55.

Mancho, C., Sainz, Á., García-Sancho, M., Villaescusa, A. and Rodríguez-Franco, F. 2011. Evaluation of perinuclear antineutrophilic cytoplasmic antibodies in sera from dogs with inflammatory bowel disease or intestinal lymphoma. Am. J. Vet. Res. 72(10), 133-137.

Mancho, C., Sainz, Á., García-Sancho, M., Villaescusa, A., Tesouro, M.A. and Rodríguez-Franco, F. 2010. Detection of Perinuclear Antineutrophil Cytoplasmic Antibodies and Antinuclear Antibodies in the Diagnosis of Canine Inflammatory Bowel Disease. J. Vet. Diagn. Invest. 22(4), 553-558.

Moser, K., Mitze, S., Teske, E., von Bomhard, W. and Stockhaus, C. 2018. Correlation of clinical, diagnostic and histopathological parameters in dogs with chronic lymphocytic-plasmacytic enteropathy. Tierarztl. Prax. Ausg. K. Kleintiere. Heimtiere. 46(1), 15-20.

Murphy, K.F., German, A.J., Ruaux, C.G., Steiner, J.M., Williams, D.A. and Hall, E.J. 2003. Fecal a1Proteinase Inhibitor Concentration in Dogs with Chronic Gastrointestinal Disease. Vet. Clin. Pathol. 32(2), 67-72.

Ogawa, M., Osada, H., Hasegawa, A., Ohno, H., Yanuma, N., Sasaki, K., Shimoda, M., Shirai, J. and Kondo, H. 2018. Effect of interleukin-1 $\beta$ on occludin mRNA expression in the duodenal and 
colonic mucosa of dogs with inflammatory bowel disease. J. Vet. Intern. Med. 32(3), 1019-1025.

Ohta, H., Sunden, Y., Yokoyama, N., Osuga, T., Lim, S.Y., Tamura, Y., Morishita, K., Nakamura, K., Yamasaki, M. and Takiguchi, M. 2014. Expression of apical junction complex proteins in duodenal mucosa of dogs with inflammatory bowel disease. Am. J. Vet. Res. 75(8), 746-751.

Okanishi, H., Hayashi, K., Sakamoto, Y., Sano, T., Maruyama, H., Kagawa, Y. and Watari, T. 2013. NOD2 mRNA expression and NFkappaB activation in dogs with lymphocytic plasmacytic colitis. J. Vet. Intern. Med. 23, 439-444.

Omori, M., Maeda, S., Igarashi, H., Ohno, K., Sakai, K., Yonezawa, T., Horigome, A., Odamaki, T. and Matsuki, M. 2017. Fecal microbiome in dogs with inflammatory bowel disease and intestinal lymphoma. J. Vet. Sci. 79(11), 1840-1847.

Osada, H., Ogawa, M., Hasegawa, A., Nagai, M., Shirai, J., Sasaki, K., Shimoda, M., Itoh, H., Kondo, H. and Ohmori, K. 2016. Expression of epithelial cell-derived cytokine genes in the duodenal and colonic mucosae of dogs with chronic enteropathy. J. Vet. Sci. 79(2), 393-397.

Otoni, C.C., Heilmann, R.M., García-Sancho, M., Sainz, A., Ackermann, M.R., Suchodolski, J.S., Steiner, J.M. and Jergens, A.E. 2018. Serologic and fecal markers to predict response to induction therapy in dogs with idiopathic inflammatory bowel disease. J. Vet. Intern. Med. 32(3), 999-1008.

Redfern, A., Suchodolski, J. and Jergens, A. 2017. Role of the gastrointestinal microbiota in small animal health and disease. Vet. Rec. 181(14), 370-370.

Rossi, G., Pengo, G., Caldin, M., Piccionello, A.P., Steiner, J.M., Cohen, N.D., Jergens, A.E. and Suchodolski, J.S. 2014. Comparison of microbiological, histological, and immunomodulatory parameters in response to treatment with either combination therapy with prednisone and metronidazole or probiotic VSL\#3 strains in dogs with idiopathic inflammatory bowel disease. PLoS. One 9(4), 1-14.

Rubio, C.P., Martínez-Subiela, S., Hernández-Ruiz, J., Tvarijonaviciute, A., Cerón, J.J. and Allenspach, K. 2017. Serum biomarkers of oxidative stress in dogs with idiopathic inflammatory bowel disease. Vet. J. 221, 56-61.

Sattasathuchana, P., Allenspach, K., Lopes, R., Suchodolski, J.S. and Steiner, J.M. 2017. Evaluation of Serum 3-Bromotyrosine Concentrations in Dogs with Steroid-Responsive Diarrhea and Food Responsive Diarrhea. J. Vet. Intern. Med. 31(4), 1056-1061.
Sattasathuchana, P., Grützner, N., Lopes, R., Guard, B.C., Suchodolski, J.S. and Steiner, J.M. 2015. Stability of 3-bromotyrosine in serum and serum 3-bromotyrosine concentrations in dogs with gastrointestinal diseases. BMC Vet. Res. 11(1), 1-7.

Sattasathuchana, P., Thengchaisri, N., Suchodolski, J.S., Lidbury, J.A. and Steiner, J.M. 2019. Analytical validation of fecal 3-bromotyrosine concentrations in healthy dogs and dogs with chronic enteropathy. J. Vet. Diagn. Invest. 31(3), 434-439.

Schnyder, M., Oevermann, A., Doherr, M., Luckschander, N., Zurbriggen, A. and Burgener, I. 2018. Dysregulation of toll-like receptors in dogs with chronic enteropathies. J. Inflamm. Bowel Dis. Disor. 3(1), 1-9.

Simpson, K.W. and Jergens, A. 2011. Pitfalls and Progress in the Diagnosis and Management of Canine Inflammatory Bowel Disease. Vet. Clin. Small. Anim. 41(2), 381-398.

Somu, Y., Muthusamy, V., Krishnakumr, S., Arulkumar, T., Jayalakshmi, K., Mani, S., Muthusamy, R., Mani. S. and Selvaraj, P. 2017. Technical review on inflammatory bowel disease in dogs and cats. Int. J. Environ. Sci. Technol. 6(3), 1833-1842.

Tamura, Y., Ohta, H., Yokoyama, N., Lim, S.Y., Osuga, T., Morishita, K., Nakamura, K., Yamasaki, M. and Takiguchi, M. 2014. Evaluation of selected cytokine gene expression in colonic mucosa from dogs with idiopathic lymphocytic-plasmacytic colitis. J. Vet. Sci. 76(10), 1407-1410.

Toresson, L., Steiner, J.M., Suchodolski, J.S. and Spillmann, T. 2016. Oral Cobalamin Supplementation in Dogs with Chronic Enteropathies and Hypocobalaminemia. J. Vet. Intern. Med. 30(1), 101-107.

Volkmann, M., Steiner, J.M., Fosgate, G.T., Zentek, J., Hartmann, S. and Kohn, B. 2017. Chronic Diarrhea in Dogs - Retrospective Study in 136 Cases. J. Vet. Intern. Med. 31(4), 1043-1055.

Wdowiak, M., Rychlik, A. and Kołodziejska-Sawerska, A. 2013. Biomarkers in canine inflammatory bowel disease diagnostics. Pol. J. Vet. Sci. 16(3), 601-609.

$\mathrm{Xu}$, J., Verbrugghe, A., Lourenço, M., Janssens, G.P.J., Liu, D.J.X., Wiele, T.V., Eeckaut, V., Immerseel, V.F., Maele, I.V., Niu, Y., Bosch, G., Junius, G., Wuyts, B. and Hesta, M. 2016. Does canine inflammatory bowel disease influence gut microbial profile and host metabolism? BMC Vet. Res. 12(1), 1-10. 